\title{
THE ASSOCIATION OF DUST AND H I GAS
}

\author{
E. BRAUNSFURTH \\ MPI für Radioastronomie, Bonn, Germany \\ and \\ K. ROHLFS \\ MPI für Radioastronomie, Bonn, Germany \\ and \\ Astronomy Program, University of Maryland, Md. 20742, U.S.A.
}

\begin{abstract}
The hypothesis that interstellar dust is associated with cold gas is advanced. To test this, such observational features of the gas that depend predominantly on the cold gas have to be isolated. Two such features are discussed, the $21-\mathrm{cm}$ line strength in absorption and the intensity of narrow emission line components in intermediate galactic latitudes. It is shown that both features have a good correlation with the dust extinction. Various measurements of the gas to dust ratio are collected and some implications for the interstellar medium are discussed.
\end{abstract}

The relative abundance of atomic hydrogen gas vs. dust grains has been a matter of controversy for many years. In most theoretical investigations Lilley's (1955) value of $\varrho_{\mathrm{H}} / \varrho_{d}=100$ has been used, but there is little observational evidence that this number is a constant. Already many years ago Bok (1953) remarked that this ratio may well vary from region to region. Indeed several authors have cast severe doubts on the assumption that an average ratio $\varrho_{\mathrm{H}} / \varrho_{d}$ has much physical meaning. Recently Wesselius and Sancisi (1971) showed quite conclusively that no general relation exists between $N_{\mathrm{H}}$ and $A_{V}$ that applies to all regions. On the other hand, just as convincing evidence can be found in the investigations of the very same authors that $N_{\mathrm{H}}$ and $A_{V}$ are correlated, if we restrict the investigations to selected regions. These relations may, however, vary from region to region. Some heuristic arguments may indicate the reasons for this variation and show what to do if one wants to find a relation applicable everywhere.

If a dust grain interacts with the interstellar gas, the growth rate of the grain radius is $\mathrm{d} a / \mathrm{d} t \propto T^{-\frac{1}{2}}$ (Wickramasinghe, 1967, Equation (6.7)) in an isobaric medium. According to Spitzer (1968, p. $181 \mathrm{ff}$ ) and Field et al. (1969) the interstellar medium does fulfill this condition, and therefore the dust grains will grow predominantly in low temperature (high density) regions. If the sticking probability of the atoms on the grain surface increases with decreasing temperature this tendency will even be stronger. To obtain information pertaining to dust formation, we therefore should compare the gas content of such cool regions with the dust extinction.

Observations of the 21-cm line in emission, however, include a fair amount of hot gas as well, and very little dust, if any, should be expected to be associated with this hot gas, if our working hypothesis is right. Therefore the observed ratio $N_{\mathrm{H}} / A_{V}$ will be too large by an amount that depends on the ratio of the hot and the cold gas along

Greenberg and Van de Hulst (eds.), Interstellar Dust and Related Topics, 231-235.

All Rights Reserved. Copyright (C) 1973 by the IAU. 
the line of sight. Means thus have to be found to distinguish observationally the contribution of the cold gas from that of the hot.

(1) Cold, dense gas has a large optical depth for $21-\mathrm{cm}$ line radiation $\left(\tau \propto N_{\mathrm{H}} / T\right)$ and thus such regions will be detectable in $21-\mathrm{cm}$ absorption profiles. A comparison of the 21-cm absorption and the dust extinction has been made by Rohlfs (1971). The 21-cm absorption data were taken from Radhakrishnan et al. (1972) and mainly extragalactic sources have been used to make sure that all neutral hydrogen gas in the

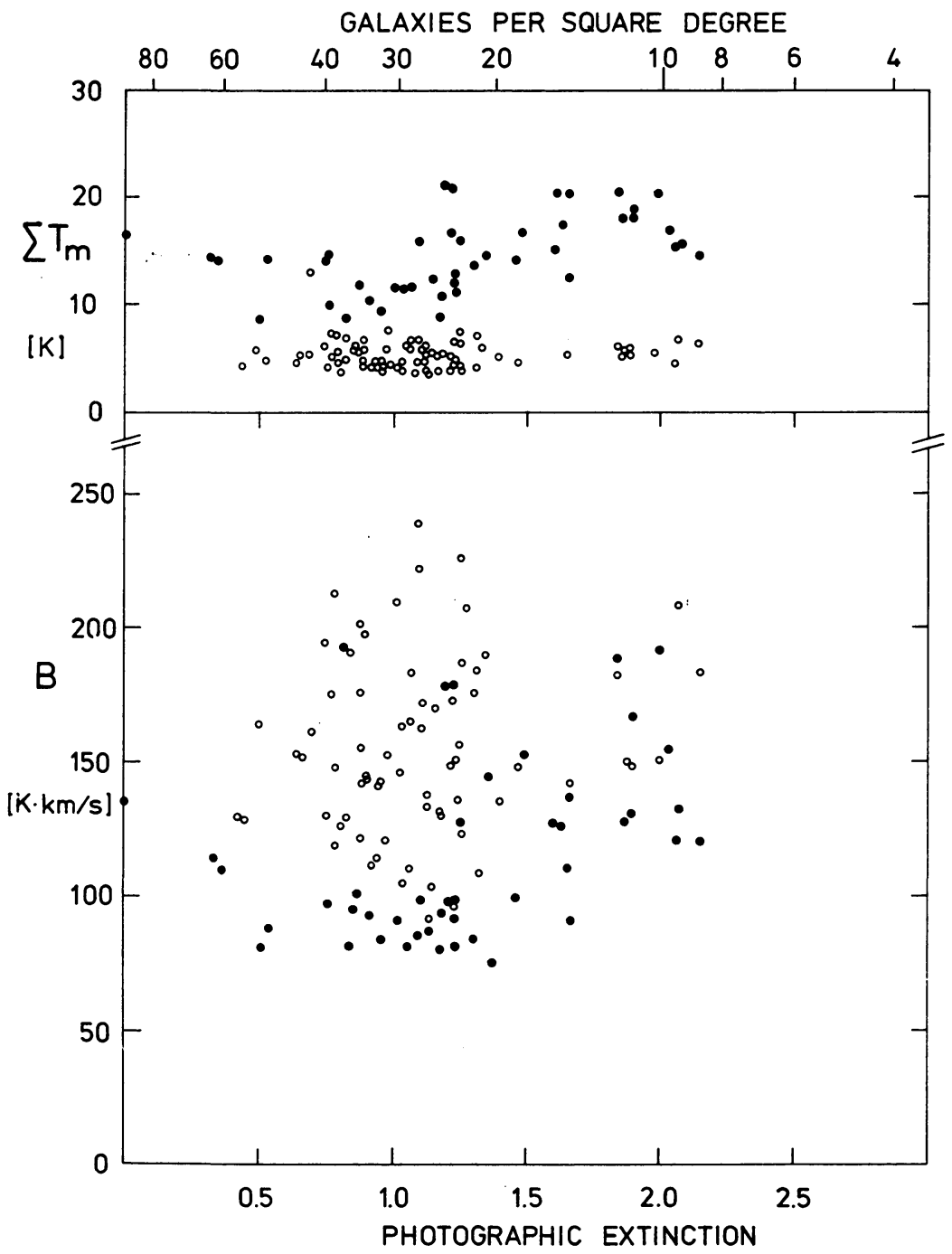

Fig. 1. Comparison of the peak brightness temperature (upper diagram) and the profile area (lower diagram) of emission line profile components with the photographic extinction in the same direction for $b^{\mathbf{I I}}=30^{\circ}$. The extinction is inferred from counts of galaxies averaged over 6 square degrees in latitude, the counts are given at the top of the diagram. Line profile components with dispersions of $10<\sigma<15 \mathrm{~km} \mathrm{~s}^{-1}$ are marked $\bigcirc$, those with $1<\sigma<4 \mathrm{~km} \mathrm{~s}^{-1}$ are marked $\bullet$. 
line of sight will be in front of the source. The optical extinction is taken from observations of OB stars with $z>500 \mathrm{pc}$ in directions close to the sources. Although sources from quite different directions in the sky were used, and even several low latitude objects were included, there is a strong correlation between the $21-\mathrm{cm}$ atomic hydrogen absorption and the visual extinction. One relation fits all data, irrespective of which area in the sky they come from. The slope of this mean relation gives $\varrho_{H} / \varrho_{d} \approx 50$. This value depends somewhat on the assumed grain model and spin temperature of the gas.

(2) Another attempt to separate the cold gas in the line of sight from the contribution of the hot gas has been made with the help of the intermediate latitude $21-\mathrm{cm}$ emission line survey of Grahl et al.(1968). The line profiles have been separated into Gaussian components. Usually a wide component with a dispersion $\sigma \approx 12 \mathrm{~km} \mathrm{~s}^{-1}$ and a narrow one with $\sigma \approx 3-4 \mathrm{~km} \mathrm{~s}^{-1}$ were found. Mebold (1972) showed that the wide component is distributed fairly uniformly through space and probably corresponds to the high temperature component of the gas. We therefore have compared the narrow components with extinction data taken from galaxy counts by Shane and Wirtanen (1967) according to the compilation by Kiang (1968). Avoiding the region $-30^{\circ}<l<30^{\circ}$ towards the galactic center, we obtained the results given in Table I for the correlation coefficients. Nonparametric Spearman ranked correlation coefficients were used to avoid problems which stem from the distribution of the data points, but both the common product-moment correlation coefficient and Kendall's ranked correlation coefficients gave essentially identical results. Thus the dust extinction is correlated mainly with narrow emission line profiles and hardly at all with wide ones. Quite similar results concerning the correlation of neutral hydrogen gas and dust extinction have been obtained for the intermediate galactic latitude survey of Takakubo et al. (1966). The details of these investigations will be reported elsewhere.

TABLE I

\begin{tabular}{|c|c|c|c|}
\hline & & $\begin{array}{l}\text { Narrow compon. } \\
1<\sigma<4\end{array}$ & $\begin{array}{l}\text { Wide compon. } \\
10<\sigma<15\end{array}$ \\
\hline$B$ vs $A_{p g}$ & $\begin{array}{l}\text { corr. coef. } \\
\text { probabil. } P\end{array}$ & $\begin{array}{l}0.42 \pm 0.12 \\
0.002\end{array}$ & $\begin{array}{l}0.07 \\
0.38\end{array} \pm 0.23$ \\
\hline$T_{\max }$ VS $A_{p g}$ & $\begin{array}{l}\text { coor. coef. } \\
\text { probabil. } P\end{array}$ & $\begin{array}{l}0.47 \pm 0.13 \\
0.0005\end{array}$ & $\begin{array}{l}0.10 \pm 0.17 \\
0.28\end{array}$ \\
\hline
\end{tabular}

In Table II results for the gas/dust ratio are collected. We have always given three quantities $B / A_{V}, N_{\mathrm{H}} / A_{V}$ and $\varrho_{\mathrm{H}} / \varrho_{d}$. Each of these contains all the information that the other has. But $B / A_{V}$ gives the ratio of two observational quantities, and no theoretical assumption enters into its determination $\left(B=\int T_{b}(V) d V\right.$ in $\left.\mathrm{K} \cdot \mathrm{km} \mathrm{s}^{-1}\right)$. The derivation of $N_{\mathbf{H}}$ involves an assumption about the optical depth of the gas, while $\varrho_{\mathrm{H}} / \varrho_{d}$ in addition requires a dust grain model. Here Lilley's (1955) model $\left(a=3 \times 10^{-5} \mathrm{~cm}\right.$, 
$Q=2, \varrho_{d}=1 \mathrm{~g} \mathrm{~cm}^{-3}$ ) was used. The range of values in the last column is due to different assumptions about the optical depth of the line radiation. Although the values given must be regarded as very uncertain and the possibility exist that both the hydrogen self absorption and the narrow emission line component underestimate the hydrogen content somewhat, we believe that the range is due to real differences and not to observational selection. A question of some importance is then, what conclusions concerning the chemical composition of the interstellar medium can be drawn from this. It should be remembered that the observations discussed here refer to atomic hydrogen only; hydrogen in any form different from this remains undetected here. If the usual values $X=0.63, Y=0.36, Z=0.014$ (Allen, 1963) for the abundance of hydrogen, helium and heavy elements by weight are adopted, and if it is assumed that all heavy elements are locked up in the dust grains, then $\varrho_{H} / \varrho_{d}=45$. Values smaller than this can only be obtained if some of the hydrogen is in a form other than atomic. At most $S=0.00083$ hydrogen by weight can be locked on the grains as $\mathrm{H}_{2} \mathrm{O}$-ice if the standard oxygen abundance is adopted. This would require that all oxygen is used up in this. The only other alternative is molecular hydrogen.

TABLE II

Gas-to-Dust Ratio according to different methods

\begin{tabular}{|c|c|c|c|c|}
\hline Source & $\begin{array}{l}\text { General high latitude } \\
\text { medium } \\
\text { (Sturch 1969) }\end{array}$ & $\begin{array}{l}21-\mathrm{cm} \text { abs. } \\
\text { (Rohlfs 72) }\end{array}$ & $\begin{array}{l}21-\mathrm{cm} \text { self } \\
\text { absorption } \\
\text { (Knapp 72) }\end{array}$ & $\begin{array}{l}\text { Narrow em. line } \\
\text { components (this paper) }\end{array}$ \\
\hline$B / A_{v}$ & $600-1100$ & 300 & 32 & 40 \\
\hline$N_{\mathrm{H}} / A_{v}$ & $1.1 \times 10^{21}-2 \times 10^{21}$ & $5.5 \times 10^{20}$ & $6 \times 10^{19}$ & $1.3 \times 10^{20}-7.3 \times 10^{19}$ \\
\hline$\varrho_{H} / \varrho_{d}$ & $100-180$ & 50 & 5.4 & $11.5-6.6$ \\
\hline$f$ & 00 & 0 & 0.88 & $0.74-0.85$ \\
\hline
\end{tabular}

The last line in Table II gives the fractional abundance of molecular hydrogen computed by

$$
f=\frac{2 n_{2}}{n_{2}+2 n_{2}}=1-\frac{Z}{X} \frac{\varrho_{\mathrm{H}}}{\varrho_{d}} .
$$

If this amount of molecular hydrogen is not observed, either as free gas or as a coating on the grains, then the only alternative is a varying chemical composition of the interstellar medium.

\section{References}

Allen, C. W.: 1963, Astrophysical Quantities, 2nd ed., Athlone Press, London.

Bok, B. J.: 1953, in J. M. Burgers and H. C. van de Hulst (eds.), 'Gas Dynamics of Cosmic Clouds', IAU Symp. 2, 221.

Field, G. B., Goldsmith, D. W., and Habing, H. J.: 1969, Astrophys. J. 155, L149.

Grahl, B. H., Hachenberg, O., and Mebold, U.: 1968, Beitr. Radioastronomie 1, 3.

Kiang, T.: 1968, Dunsink Obs. Publ. 1, No. 5, 109.

Knapp, G. R.: 1972, Thesis, Univ. of Maryland. 
Lilley, A. E.: 1955, Astrophys. J. 121, 559.

Mebold, U.: 1972, Astron. Astrophys. 19, 13.

Radhakrishnan, V., Brooks, J. W., Goss, W. M., Lockhart, P., Murray, J. D., and Schwartz, U. J.: 1972, Astrophys. J. Suppl. 24, 1.

Rohlfs, K.: 1971, Astron. Astrophys. 13, 46.

Shane, C. D. and Wirtanen, C. A.: 1967, Publ. Lick Obs. 22, Part 1.

Spitzer, L.: 1968, Diffuse Matter in Space, Interscience Pub. N.Y.

Sturch, C.: 1969, Astron. J. 74, 82.

Takakubo, H. and van Woerden, H.: 1966, Bull. Astron. Inst. Neth. 18, 488.

Wesselius, P. R. and Sancisi, R.: 1971, Astron. Astrophys. 11, 246.

Wickramasinghe, N. C.: 1967, Interstellar Grains, Chapman and Hall Ltd., London. 